\title{
e-Migrinter
}

$7 \mid 2011$

Réflexions croisées sur les migrations en Afrique de l'Ouest

\section{La circulation migratoire des mineurs dits « non accompagnés » entre le Sénégal et l'Espagne}

Daniel Senovilla Hernàndez et Elh Ousmane Cissé

\section{OpenEdition}

\section{Journals}

Édition électronique

URL : https://journals.openedition.org/e-migrinter/858

DOI : 10.4000/e-migrinter.858

ISSN : 1961-9685

Éditeur

UMR 7301 - Migrinter

Édition imprimée

Date de publication : 1 septembre 2011

Pagination : 6-16

ISSN : 1961-9685

\section{Référence électronique}

Daniel Senovilla Hernàndez et Elh Ousmane Cissé, « La circulation migratoire des mineurs dits «non accompagnés » entre le Sénégal et l'Espagne », e-Migrinter [En ligne], 7| 2011, mis en ligne le 07 septembre 2017, consulté le 20 mai 2021. URL : http://journals.openedition.org/e-migrinter/858 ; DOI : https://doi.org/10.4000/e-migrinter.858 


\title{
DOSSIER
}

\section{$1^{\text {ère }}$ partie : Trajectoires migratoires et migrants « acteurs »}

\author{
La circulation migratoire des mineurs \\ dits « non accompagnés » entre le Sénégal et l'Espagne
}

\author{
Elh Ousmane Cissé \& Daniel Senovilla Hernández
}

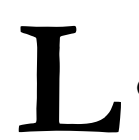

'idée d'écrire un article commun sur la circulation migratoire des mineurs sénégalais vers l'Espagne a émergé à la suite d'une réflexion collective des doctorants de Migrinter sur les mobilités en Afrique de l'Ouest. A cette occasion, la question des mineurs nous est apparue particulièrement pertinente à partir de la prise en compte des stratégies déployées aussi bien au niveau du pays de départ que de celui d'arrivée.
La plupart des recherches sur le sujet s'intéressent surtout aux politiques mises en place par les pays européens pour la gestion de ces flux. Certaines s'intéressent aux modèles d'accueil développés par les pays européens pour mieux maîtriser l'accueil des mineurs étrangers, mais il existe un véritable déficit de travaux scientifiques sur les stratégies mises en place par les acteurs de la migration dans les pays d'origine pour faciliter le départ des candidats.

Le Sénégal est un pays de tradition migratoire ancienne vers les pays du Nord. Cette mobilité concernait «les gens du fleuve » originaires des villages de la vallée du fleuve Sénégal particulièrement touchée par la grande sécheresse des années 1960. Cette période de sécheresse a coïncidé avec la période d'appel de main-d'œuvre de la France. "Même après l'avènement des indépendances, l'accord multilatéral ratifié notamment avec la Fédération du Mali en 1960, puis avec le Sénégal en 1961 après la rupture de la Fédération établissant la libre circulation des personnes entre États membres de la communauté: les Sénégalais, les Maliens et les Mauritaniens n'avaient besoin que d'une pièce d'identité nationale pour entrer en France 》 (Mouhamadou Lamine Ndiaye, 1992). Ces hommes, souvent jeunes, qui partaient en France espéraient trouver des conditions économiques leur permettant de gagner de l'argent afin d'aider leurs familles restées au pays. Ainsi, les investissements de ces migrants consistaient 
souvent à satisfaire les besoins de consommation des leurs. Même si la France constituait une destination privilégiée des migrants Sénégalais dans les années 1960, des pays ouest africains comme la Côte d'Ivoire ou le Burkina Faso et d'Afrique Centrale (Gabon, Cameroun, ex Zaïre et République du Congo) restaient les destinations de la majorité des migrants du fait de liens historiques (colonisation) ou géographiques (proximité) avec le Sénégal (Traoré, 1994).

L'émigration sénégalaise a cependant connu une diversification aussi bien au niveau des pays d'accueil que des lieux d'origine des migrants à l'intérieur du Sénégal. Aujourd'hui, l'émigration est devenue un phénomène national dans la mesure où les candidats à l'émigration viennent de toutes les régions du Sénégal. Cette diversification des zones de départ a ensuite entraîné une diversification des profils des migrants. C'est ainsi que des femmes et des enfants mineurs ont quitté le Sénégal pour rejoindre, souvent par voie aérienne, leurs maris ou parents installés en France ou en Europe. Aujourd'hui, on constate également le départ en Europe d'enfants mineurs non accompagnés hors du cadre du regroupement familial avec pour objectif de travailler ou de bénéficier d'une formation qui leur permettrait de gagner de l'argent afin d'aider leur famille. Ces mineurs ont des parcours migratoires par étapes et des moyens de transports souvent divers. C'est le cas des mineurs que nous avons interrogés dans le quartier de Hann à Dakar et qui, pour la plupart d'entre eux, ont embarqué sur des pirogues pour rejoindre l'Europe par voie maritime via les Îles Canaries. Certains d'entre eux sont amenés au cours de leur voyage à transiter par la Mauritanie ou le Maroc.

En Espagne, pays devenu aujourd'hui l'une des principales destinations de travailleurs migrants dans le monde, les arrivées de mineurs qui ne sont pas accompagnés de leurs parents ou de leurs tuteurs légaux ont commencé timidement dans les années 1990. Jusqu'au début des années 2000, la très grande majorité des mineurs migrants arrivant en Europe était d'origine marocaine $90 \%$ des mineurs non accompagnés accueillis en Espagne étaient marocains). Ce n'est qu'à partir de l'année 2005 qu'ont commencé les arrivées de mineurs originaires d'Afrique de l'Ouest, en particulier du Sénégal, aux Îles Canaries. En 2006, 931 mineurs dits «non accompagnés » sont arrivés aux Îles Canaries, presque la moitié (453) étant de nationalité sénégalaise, sur un total de plus de 31000 immigrés arrivés dans l'archipel par voie maritime ${ }^{1}$. En 2007 et 2008, les chiffres ont légèrement baissé mais les mineurs non accompagnés comptabilisés sont estimés à 750 par an, avec une présence toujours forte des originaires du Sénégal ${ }^{2}$. À partir de 2009, un ensemble de facteurs (entre autres, l'impact de la crise économique en Espagne mais aussi le durcissement des mesures de contrôle migratoire notamment dans les différents pays d'origine) ont provoqué un fort ralentissement de ce type de migration et les arrivées de pirogues sont aujourd'hui nettement plus modestes. A la différence de 2006 plus de 31.000 personnes étaient arrivées par voie maritime aux Canaries, seulement 2246 personnes sont arrivées par cette voie en $2009^{3}$.

\footnotetext{
1 Source: "Dictamen de la Comisión de Estudio de Jóvenes y Menores de Canarias", Journal officiel du Parlement des îles Canaries du 28 mars 2007, p. 24.

2 Un article de presse qui cite les chiffres du Gouvernement régional des îles Canaries signale l'arrivée de 752 mineurs non accompagnés en 2007 et 754 en 2008. Voir "Canarias quiere devolver al Estado la competencia sobre niños sin papeles", article signé par T. Bárbulo y J. Naranjo, quotidien «El País» du 29 janvier 2009. Voir aussi le Journal officiel du Sénat espagnol, série I, n 294, du 3 aout 2009, p. 151.

3 Source : Journal officiel du Congres espagnol, série D, n 388, du 7 mai 2010, p. 538.
} 


\section{Le rôle des différents acteurs impliqués dans le processus d'émigration}

L'émigration des mineurs sénégalais par voie maritime implique plusieurs acteurs qui jouent chacun un rôle important dans le processus de départ. Ces acteurs sont les mineurs eux-mêmes, mais aussi les familles et d'autres personnes adultes comme les organisateurs des départs, notamment les patrons d'embarcation, les intermédiaires et les personnes qui se chargent de l'accueil des mineurs une fois qu'ils arrivent en Europe. Pour analyser le rôle des différents acteurs, une enquête a été menée à Dakar en 2009 auprès de quinze mineurs, cinq mères de famille et trois passeurs (Dakar, quartier de Hann, mars - avril 2009) et à Valence en 2010 à travers quatre entretiens avec trois mineurs sénégalais et un adulte qui accueillait des Sénégalais ayant émigré par voie maritime après leur libération des centres de rétention (Valence, Espagne, décembre 2010).

\section{Le rôle du mineur migrant en tant} qu'acteur de son parcours migratoire

Certains mineurs réfléchissent seuls au choix de leur départ du Sénégal en pirogue, comme c'est le cas de plusieurs d'entre eux que nous avons rencontrés dans le quartier à Dakar (la majorité ne vivait d'ailleurs plus avec les parents). Beaucoup sont originaires des campagnes sénégalaises et ont l'habitude de travailler dans les grandes villes à la fin de la période de récolte. Lorsqu'ils sont en ville, ces mineurs travaillent souvent dans le commerce informel. Cette migration temporaire de travail leur permet de faire des économies et d'aider leurs parents restés à la campagne. Lors de nos entretiens, certains mineurs nous ont expliqué que leur décision de partir relevait d'une stratégie individuelle. Ces enfants avaient peur d'en informer leurs parents du fait du risque élevé du voyage en pirogue et donc du potentiel refus de leur famille. $\mathrm{Ce}$ risque migratoire était généralement connu des familles car "la presse et les médias dans leur ensemble font largement état des accidents. Parallèlement, des campagnes de sensibilisation, initiées par les pays européens et menées par les ONG locales on internationales, relaient les récits de ceux qui n'ont pas réussi à atteindre le pays soubaité ou de ceux qui ont été "réadmis », rapatriés le plus souvent par les autorités espagnoles» (Gonin et Robin, 2009).

«Mes parents n'étaient pas au courant de mon départ car je me disais qu'ils ne vont pas me laisser prendre la pirogue. Ils sont au courant de cette émigration et ils savent qu'il y a des morts. C'est pourquoi j'avais peur mais je n'ai aucun problème avec eux» (M.B, 30/12/10, Valence)

D'autres mineurs, qui ont l'habitude de travailler comme apprentis pêcheurs dans les pirogues, étaient chargés d'amener les candidats à l'émigration et n'ont donc pas été soumis à l'obligation de payer leur voyage. Généralement, c'est cette situation d'opportunité qui les a poussés à prendre la décision de partir. Certains d'entre eux ont tenu leurs parents au courant de leur décision, mais d'autres ont préféré attendre leur arrivée en Espagne pour informer leurs parents.

"Nous qui avions l'habitude d'aller en mer
avec la pirogue pour des campagnes de pêche
nous n'avions pas payé. Les autres qui ne
faisaient pas partie de l'équipage payaient
aux propriétaires de la pirogue une somme
mais je ne sais pas combien" (M.G,
16/04/09, Hann).

Enfin, d'autres encore qui veulent partir mais n'ont pas les moyens de payer le voyage fréquentent la plage en espérant trouver une place dans une pirogue. Les stratégies de ces enfants consistent donc à surveiller les pirogues pendant la préparation des départs ou à se cacher sous les marchandises selon les témoignages des organisateurs des voyages que nous avons rencontrés (figure $\mathrm{n}^{\circ} 1$ ).

Il est important de signaler que ces enfants sont partis de leur propre gré et ne 
sont pas victimes de maltraitances physiques de la part de leurs parents comme ce fut le cas de certains mineurs en provenance de pays comme le Maroc ou l'Europe de l'Est. C'est seulement la peur que leurs parents n'adhèrent pas à leur projet qui les a poussés à prendre la décision de partir sans informer leur famille. Si certains mineurs avaient peur de mettre au courant leur famille de leur projet migratoire, d'autres ont, au contraire, été mandatés par des membres de leur famille pour prendre la voie de l'émigration maritime.

Figure $\mathbf{n}^{\circ} 1$ : Des mineurs aidant les pêcheurs à préparer leurs filets de pêche sur la plage de Hann.

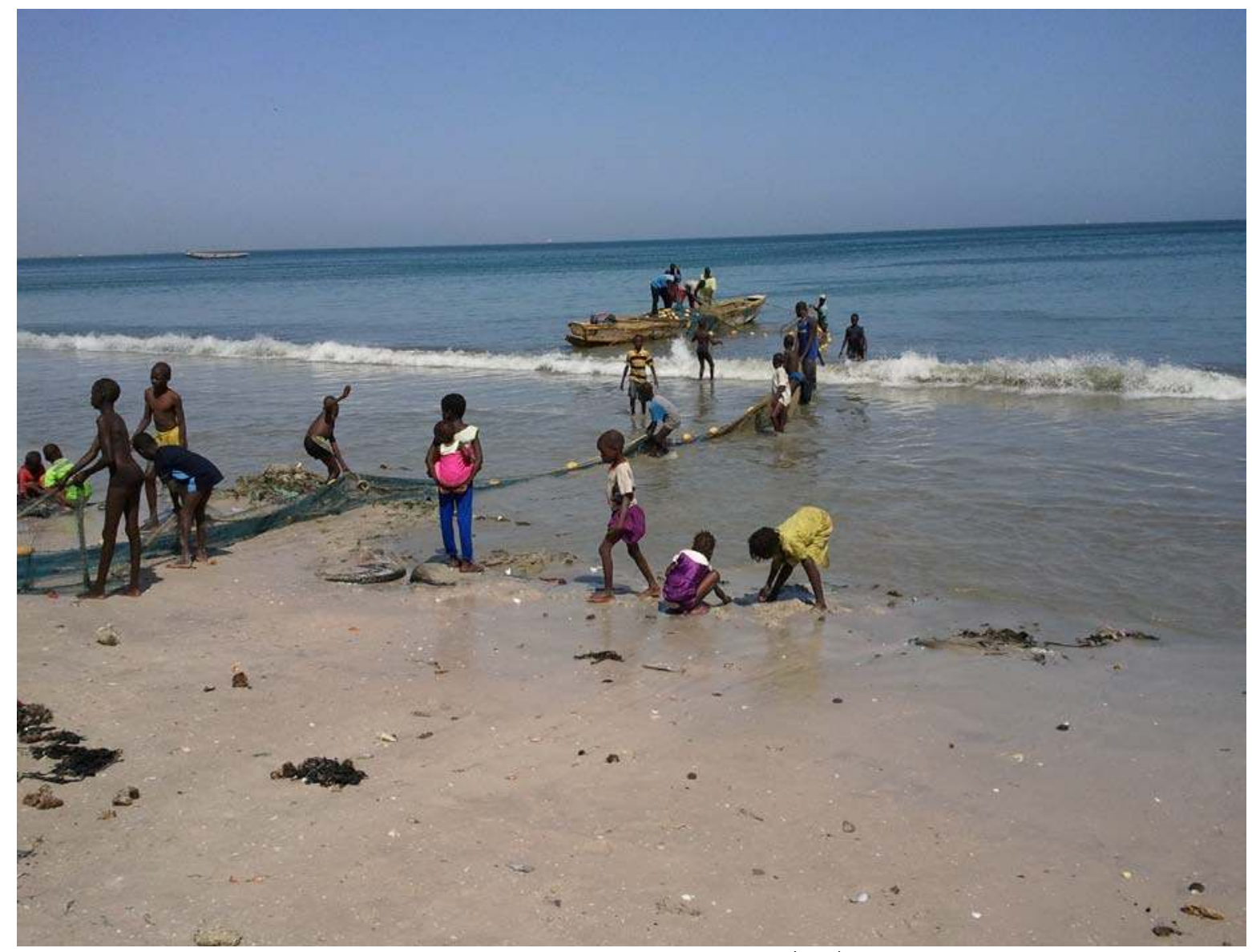

Source: Cissé Elh Ousmane, Dakar, 30/03/2009.

\section{Le rôle de la famille dans la prise} de décision migratoire

Les entretiens avec certaines mères de familles ont mis en évidence l'importance de leur rôle dans le projet d'émigration de leurs enfants. Ces familles envoient leurs enfants en migration pour qu'ils puissent trouver du travail en Europe et apporter un revenu supplémentaire à la famille. Le plus souvent c'est la mère qui décide le départ de l'enfant, ce qui s'explique dans le contexte sénégalais actuel où de plus en plus de femmes deviennent chefs de ménage, notamment à
Dakar. Cette proportion de femmes chefs de ménage est plus importante dans les zones de pêche, principaux lieux de départ des candidats à l'émigration par voie maritime, où les hommes partent en mer et les femmes s'occupent du ménage pendant leurs absences. Certaines femmes ont envoyé leurs enfants en Europe par effet de mimétisme après le départ d'autres enfants du même quartier. Elles considèrent l'émigration en Europe comme une chance de réussite pour leurs enfants. Ces femmes que nous avons rencontrées travaillent dans le commerce, ce qui leur permet de financer une partie ou 
parfois la totalité de la somme exigée par les organisateurs des départs pour la traversée de leurs enfants vers l’Espagne (figure $n^{\circ} 2$ ).

D'autres mères n'ayant pas la possibilité de réunir la somme exigée par les organisateurs des départs ont recours à l'emprunt ou à la vente de leurs effets personnels, tels que leurs bijoux, pour financer le départ de leur enfant. Ces stratégies utilisées par les mères de famille ont eu des influences sur le parcours migratoire de leurs enfants. Ces mères, contraintes de payer la dette contractée, et averties du fait que les mineurs ne peuvent pas travailler en Espagne, ont demandé à leurs enfants de se déclarer majeurs. Cette pratique permet en effet aux mineurs de travailler en Espagne et d'envoyer de l'argent à leurs mères pour qu'elles puissent honorer la dette liée au départ.

"Avant de venir en Espagne, ma mère m'a demandé de dire aux autorités que j'ai plus de 18 ans sinon je ne pourrais pas travailler et l'aider surtout qu'elle s'est endettée pour payer mon départ» (extrait entretien réalisé en Espagne).

Figure $\mathbf{n}^{\circ} 2$ : Des femmes vendeuses de poisson au Marché de Hann Plage.

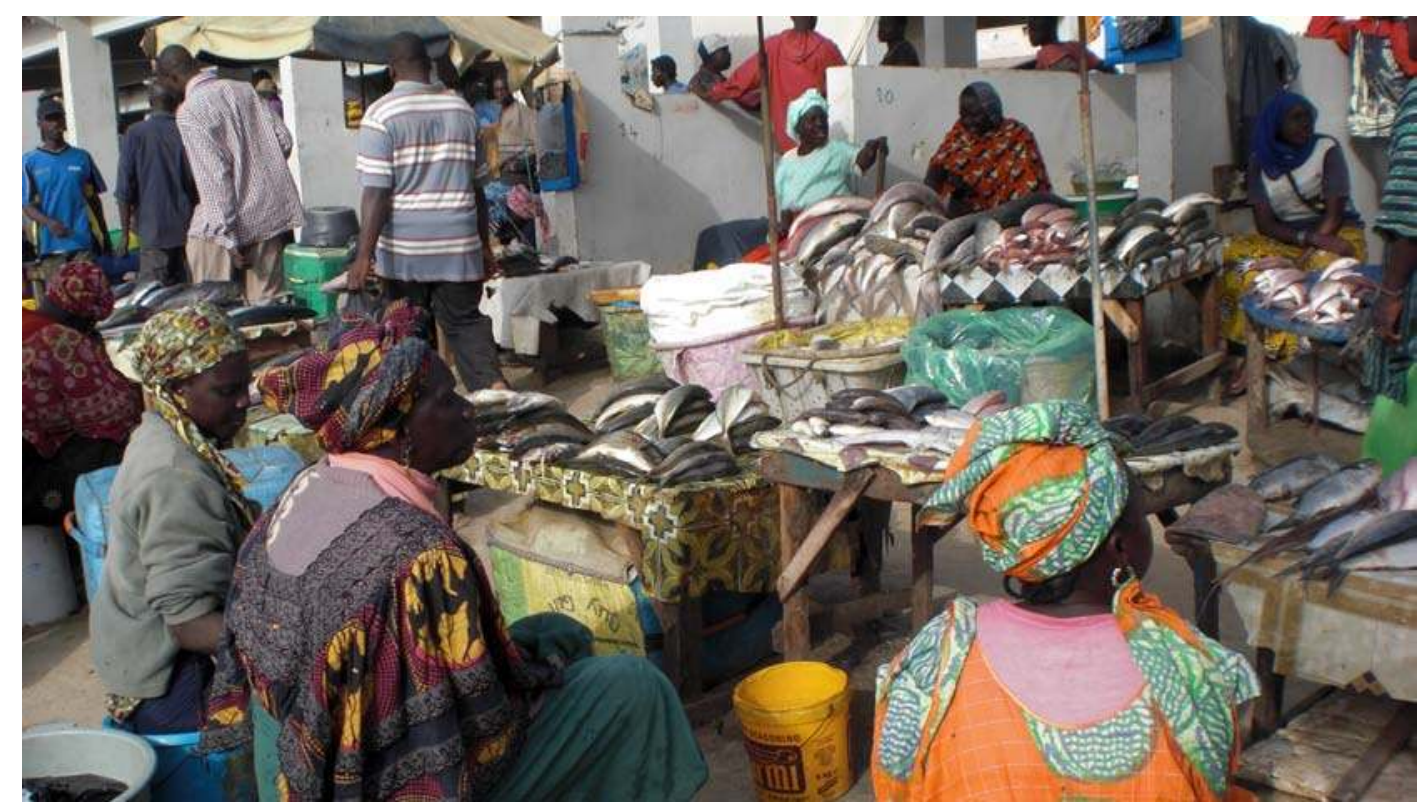

Source : Cissé Elh Ousmane, Dakar, 2009.

Cette tendance des mères à envoyer leur enfant est liée au fait que ces femmes cherchent à protéger leur place dans des familles nombreuses et souvent polygames. La polygamie entraîne des rivalités entre les femmes, aussi le fait d'envoyer son enfant en Espagne pour qu'il travaille et aide la famille permet une revalorisation du statut de la mère vis-à-vis des co-épouses.

"Nos maris sont polygames et nous, les épouses nous nous livrons à une véritable compétition. Ainsi la première qui parvient à faire partir un fils est considérée comme la meilleure épouse ${ }^{4}$.

Même si les mères des mineurs ont joué un rôle déterminant dans le processus de départ de leurs enfants, d'autres personnes adultes ont également contribué à la mise en place des réseaux migratoires qui ont facilité l'émigration par voie maritime.

\footnotetext{
${ }^{4}$ Abi Samb, vice-présidente du Collectif des Femmes pour la lutte contre l'immigration clandestine de Thiaroye-Sur-Mer.
} 


\section{Le rôle des patrons d'embarcation et des intermédiaires dans l'organisation des départs}

Le recrutement des candidats au Sénégal est soit effectué directement par les propriétaires des pirogues dans les quartiers de départ soit via l'implication d'autres personnes chargées de recruter des candidats hors des zones de départ. Les intermédiaires monnayent une contrepartie financière ou des places dans les pirogues. Certains intermédiaires sont originaires du quartier mais la plupart viennent d'autres régions comme Louga, Kolda, Saint-Louis et Diourbel. Selon nos informateurs, ces derniers ont beaucoup contribué à la diffusion de la pratique d'émigration par voie maritime à Hann en fournissant de nombreux candidats au départ.

"J'ai organisé six départs vers les Iles Canaries. Pour y arriver, je faisais appel à d'autres personnes qui étaient chargées de me chercher des clients. Ces personnes étaient payées entre 25000 et 50000 Francs CFA soit 35 à 70 euros. Ils étaient au nombre de 4 dont 2 qui babitent à Hann et pour ce qui est des 2 autres l'un babite à Louga l'autre à Diourbel. » (A. G., le 08/04/2009).

Ces intermédiaires amènent les candidats chez le propriétaire de la pirogue qui prend leurs coordonnées ainsi qu'un acompte sur le prix du voyage (généralement la moitié) ; le reste étant payé le jour même du départ. Pour d'autres organisateurs, le paiement se fait intégralement le jour de l'inscription. Au moment de l'inscription, chaque candidat donne un numéro de téléphone sur lequel il sera appelé pour être informé de la date et du lieu de départ. Une fois le recrutement des candidats effectué, les propriétaires des pirogues commencent à les préparer pour le départ.

Les patrons d'embarcation se chargent de la construction ou de la réfection des pirogues. Les propriétaires de celles-ci consultent ensuite les sites météorologiques pour savoir si le temps est favorable au départ : pour A.G (propriétaire de pirogues à Hann), c'est seulement après avoir pris connaissance du temps qu'il décide du jour et du lieu de départ, ensuite communiqués aux candidats. Pour assurer la sécurité des passagers, certains organisateurs consultent des marabouts pour la préparation mystique de la pirogue. Ils peuvent aussi faire appel à un policier qui va se déplacer sur le lieu du départ pour assurer la sécurité et empêcher les personnes qui n'ont pas payé de monter dans la pirogue.

Ces organisateurs de départ recrutent aussi des pêcheurs capables de conduire la pirogue en Espagne. Ces capitaines ont l'habitude de conduire des pirogues pour des campagnes de pêche. Ils sont payés avant le départ. Ensuite ils bénéficient d'un voyage gratuit car une fois arrivés en Espagne, ils restent et tentent de trouver un travail.

\section{Une émigration facilitée par la présence d'un membre de la famille en Europe.}

Les réseaux familiaux en Europe jouent un rôle important dans l'émigration de certains mineurs sénégalais. Avant le départ, les mineurs se renseignent sur les possibilités de se faire héberger dans les pays de destination. La plupart des enfants disposent du numéro de téléphone d'une personne qu'ils doivent contacter une fois arrivés en Europe. Certains des enfants affirment qu'ils seront attendus par des membres de leur famille qui sont déjà installés en Espagne. Ces personnes sont, en général, un frère, un cousin ou une simple connaissance dans le quartier qui avait déjà pris la pirogue pour rejoindre l'Europe. C'est le cas de Nd. Y dont l'enfant est en Espagne dans un centre d'accueil pour mineurs alors que son but était de rejoindre son père qui se trouvait en Italie. Cette importance du réseau familial est plus marquée chez les jeunes qui viennent des autres régions du Sénégal (Saint Louis, Louga, Kolda, Diourbel). Un organisateur de voyage nous a informé que la plupart des gens qui partent 
dans les pirogues ne sont pas originaires de Dakar. Cette situation s'explique par le fait que les premiers émigrés venaient des campagnes sénégalaises et ont mis en place des réseaux (comme les associations villageoises) qui favorisent l'intégration des nouveaux venus. Ainsi, les gens de la campagne connaissent toujours un parent qui se trouve en Europe. En Espagne nous avons rencontré B.S, un sénégalais qui aurait accueilli plus de vingt migrants sénégalais arrivés en pirogue. Son rôle consistait à aller les chercher à la gare ou à l'aéroport et à les héberger le temps de leur trouver un logement. Ils les aidaient aussi à s'insérer dans le commerce informel car pour lui c'est le seul métier que peut faire un «sans papiers » en Espagne.

\section{La pratique du « confiage » de mineurs}

La pratique du «confiage » consiste à placer son enfant biologique, le plus souvent mineur, dans une famille adoptive. Cette pratique est répandue au Sénégal et elle touche la plupart du temps des familles dont les revenus sont modestes et ne permettent pas de subvenir aux besoins de tous ses membres. Certaines familles ont recours à cette pratique dans le but de permettre à leurs enfants de bénéficier d'une formation qu'elles ne peuvent pas leur assurer. C'est le cas des enfants qui sont confiés à des marabouts pour qu'ils puissent avoir une éducation religieuse. D'autres mineurs sont «confiés» à des professionnels, comme apprentis, pour qu'ils soient formés à un métier manuel. Ces pratiques de "confiage» se sont étendues aux stratégies migratoires de certains parents afin d'assurer le bon déroulement du voyage de leur enfant. Ces mineurs sont le plus souvent confiés à un membre de la famille ou à une connaissance qui doit également voyager. Le rôle de l'adulte « responsable » consiste à veiller à la sécurité du mineur durant la traversée et à l'aider à son arrivée en Espagne.

Parfois, cette pratique de "confiage» peut avoir des conséquences néfastes pour les mineurs. En effet, au Sénégal des enfants «confiés » se retrouvent souvent dans la rue pour faire la manche, pouvant ainsi être exposés à toutes formes de maltraitance. Aussi l'Assemblée nationale sénégalaise a-telle voté en 2010 une loi interdisant la mendicité des enfants dans les rues. Ces dysfonctionnements observés au Sénégal nous amènent à nous interroger sur la pertinence de la reconnaissance de la pratique de «confiage» une fois que le mineur migrant a quitté le territoire sénégalais : faut-il laisser la protection de l'enfant à l'adulte responsable auquel il est confié depuis son départ du Sénégal ou fautil les séparer pour éviter que l'enfant ne soit exploité par l'adulte comme on a pu l'observer pour certains mineurs confiés au Sénégal? Nous reviendrons sur cette question en analysant d'un point de vue légal les conséquences qu'impliquerait une reconnaissance de cette pratique une fois le mineur arrivé en Europe. Jusqu'à présent, les autorités espagnoles et européennes ont systématiquement séparé ces enfants des adultes à qui ils étaient «confiés». Le témoignage qui suit illustre bien cette situation :

"Mon fils était accompagné par son grand frère mais quand ils sont arrivés en Espagne son grand frère a appelé pour nous dire qu'il a été séparé de mon enfant. Lui, il est parti avec les autres membres de la pirogue et mon enfant a été amené par les espagnols je ne sais pas où. J'avais peur en ce moment car je me demandais pourquoi mon fils est séparé de son frère, je ne dormais pratiquement pas pendant des nuits. Un jour mon fils m'a appelé pour me dire qu'il est avec des enfants et qu'il est bien traité. C'est en ce moment que j'ai commencé à dormir tranquille. Je suis allé me renseigner vers d'autres mères de famille qui ont leur enfant en Espagne pour leur demander si leurs enfants avaient aussi été séparés de leur accompagnant et elles m'ont répondu que c'est normal, en Espagne les enfants doivent être isolés pour apprendre un métier. Quelques semaines plus tard mon fils m'a encore appelé pour me dire qu'il est 
en train de faire une formation. » (Y. $\mathrm{Nd}$, Pêcheur, Dakar, le 24/03/2009).

\section{La pertinence de la définition et du traitement du mineur étranger « non accompagné " par rapport aux réalités sociales qui déterminent cette circulation migratoire}

Les résultats d'une enquête réalisée sur un échantillon de plus de 300 mineurs d'origine sénégalaise arrivés aux îles Canaries entre 2006 et $2007^{5}$ confirment les résultats décrits précédemment à partir des enquêtes au Sénégal. Une majorité des mineurs sénégalais accueillis en Espagne travaillait préalablement dans le secteur de la pêche et avait fait la traversée vers les Îles Canaries sans payer le voyage, en tant que membre d'équipage de la pirogue utilisée. Ces mineurs avaient été "confiés» par leurs familles à une personne adulte qui prenait en charge leur éducation et leur apprentissage d'un métier selon les traditions de cette pratique coutumière très répandue en Afrique de l'Ouest et n'avaient donc pas voyagé seuls mais accompagnés d'un adulte responsable. D'un point de vue juridique, doit-on considérer que ces mineurs arrivés en Espagne confiés devaient être considérés comme des mineurs sous l'autorité parentale d'un adulte responsable et, en conséquence, sous la responsabilité de cette personne?

Avant d'essayer de répondre à cette question, il convient de rappeler brièvement les différentes définitions et termes utilisés dans le contexte européen pour designer le phénomène de la migration indépendante de personnes mineures. Ces termes correspondent initialement aux différentes traditions politiques de gestion des mouvements de population dans chaque pays et aux différents modèles basés sur

\footnotetext{
${ }^{5}$ Enquête Alondra réalisée par la Fondation Nouveau Soleil. Les résultats bruts de cette enquête ont été analysés lors de deux publications : Iglesias Martinez, Juan \& Legaz Torres, Francisco (2009) et, Senovilla Hernández, Daniel ; Touzenis, Kristina ; Kanics, Jyothi (2010) cf. bibliographie.
}

l'application des normes internationales régissant l'octroi du droit d'asile ou la considération classique des migrants comme main-d'œuvre.

Une distinction doit être établie, d'une part, entre les mineurs qui au cours de leur processus migratoire se retrouvent totalement seuls ou accompagnés par d'autres adultes avec qui ils n'ont pas de liens de parenté et, d'autre part, ceux accompagnés par un membre de leur famille autre que leurs parents ou tuteurs légaux. Précisément, l'Observation générale $n^{\circ} 6$ du Comité des droits de l'enfant du $1^{\text {er }}$ septembre 2005 a établi une double définition qui différencie les mineurs non accompagnés (ceux qui ne sont accompagnés ni par leurs parents ou tuteurs, ni par un autre membre de la famille qui aurait pu être chargé de veiller sur l'enfant) des mineurs séparés (qui sont effectivement séparés de leurs parents ou tuteurs légaux mais qui peuvent être accompagnés par d'autres membres de la famille élargie). ${ }^{6}$

Quant aux différents instruments législatifs de l'Union européenne (Directives et règlements régulateurs de l'asile et l'immigration), ils utilisent le terme de "mineur non accompagné » et proposent une définition équivalente à celle préalablement énoncée à l'article 1 de la résolution du Conseil du 26 juin 1997 concernant les mineurs non accompagnés ressortissants de pays tiers (97/C 221/03),

\footnotetext{
6 Observation générale $\mathrm{n}^{\circ} 6$ (2005) relative au traitement des enfants non accompagnés et des enfants séparés en dehors de leur pays d'origine, CRC/GC/2005/6 du $1^{\text {er }}$ septembre 2005, alinéa 7 : "Par "enfant non accompagné" (également appelé mineur non accompagné), on entend un enfant, au sens de l'article premier de la Convention, qui a été séparé de ses deux parents et d'autres membres proches de sa famille et n'est pas pris en charge par un adulte investi de cette responsabilité par la loi ou la coutume » et alinéa 8: "Par "enfant séparé", on entend un enfant, au sens de l'article premier de la Convention, qui a été séparé de ses deux parents ou des personnes qui en avaient la charge à titre principal auparavant en vertu de la loi ou de la coutume, mais pas nécessairement d'autres membres de sa famille. Un enfant séparé peut donc être accompagné par un autre membre adulte de sa famille ».
} 
qui constitue à ce jour le seul instrument de droit communautaire spécifiquement consacré à ce phénomène: "La présente résolution concerne les ressortissants de pays tiers âgés de moins de dix-huit ans qui entrent sur le territoire des États membres sans être accompagnés d'un adulte qui soit responsable d'eux, de par la loi ou la coutume, et tant qu'ils ne sont pas effectivement pris en charge par une telle personne. La présente résolution peut également s'appliquer à des mineurs ressortissants de pays tiers qui ont été laissés seuls après être entrés sur le territoire des États membres. Les personnes auxquelles s'appliquent les deux alinéas précédents sont dénommées ci-après "mineurs non accompagnés" ».

La réglementation européenne accorde clairement donc sa préférence au terme «non accompagné » et harmonise une définition qui se fonde essentiellement sur le critère d'absence de personnes responsables de la prise en charge du mineur, conformément à la loi ou à la coutume. Mais cette définition communautaire pâtit d'un manque de précision. Elle établit tout d'abord le concept indéterminé d'«adulte responsable» sans préciser si l'on fait exclusivement référence aux titulaires de l'autorité parentale (c'est-à-dire aux parents ou tuteurs légaux) ou si cette notion peut être étendue à d'autres membres de la famille du mineur. Aussitôt après, elle précise que cet « adulte responsable » doit l'être de par la loi ou la coutume (sachant que l'on entend ici celle du pays d'origine du mineur). Nous sommes ici confrontés à un problème puisqu'il nous faudrait pouvoir connaitre et apprécier les législations et coutumes des nombreux pays d'origine d'où émigrent les mineurs pour déterminer dans chaque cas si nous nous trouvons face à un mineur «accompagné » d'un adulte responsable ou non.

Finalement, le droit espagnol ne propose aucune définition du terme " mineur étranger non accompagné » (celui utilisé par les normes). En tout cas, la Loi des étrangers du 11 décembre 2009 (article 35) fait référence de façon implicite aux personnes mineures de moins de 18 ans qui sont identifiées en situation irrégulière au moment de l'arrivée en Espagne ou sur le territoire quand elles sont soupçonnées de se trouver dans une situation de délaissement (situation définie par l'article 172 du Code Civil qui implique que les parents ou détenteurs de l'autorité parentale sont provisoirement ou définitivement incapables d'exercer leurs devoirs en tant que tels). Les mineurs qui se trouvent dans cette situation sont accueillis par les services régionaux de protection de l'enfance qui, une fois confirmées la minorité et la situation de délaissement, deviennent les tuteurs légaux de l'enfant.

Dans le cas de l'émigration des mineurs sénégalais vers les Iles Canaries, les données du terrain issues des enquêtes menées au Sénégal révèlent qu'une partie significative des enfants migrants n'affrontent pas seuls leur parcours migratoire mais souvent accompagnés par d'autres adultes auxquels ils ont été confiés par leurs parents. La question qu'on se pose à nouveau est la suivante: le système de «confiage» pratiqué dans cette région de l'Afrique peut-il et doit-il, une fois que le mineur se trouve en Espagne, être assimilé à une délégation de l'autorité parentale ? Doiton alors considérer que l'enfant « confié » est sous la responsabilité de l'adulte accompagnateur?

En répondant à cette question par l'affirmative, le traitement juridique du mineur migrant qui se trouve en Espagne change radicalement. Si l'on présume que l'adulte auquel le mineur a été confié essaie aussi de pénétrer clandestinement sur le territoire espagnol, le statut légal du mineur ne serait plus celui d'un mineur non accompagné mais équivalent à celui d'un enfant en situation irrégulière qui se trouve avec ses parents eux aussi en situation irrégulière. Selon les normes espagnoles et la pratique administrative courante, la conséquence directe de l'identification d'une personne étrangère qui essaie d'accéder 
irrégulièrement sur le territoire implique l'ouverture automatique d'un dossier d'expulsion et le placement dans un centre de rétention pendant une période qui peut se prolonger jusqu'à 60 jours.

L'article 62 de la Loi des étrangers du 11 décembre 2009 établit que les mineurs ne peuvent pas être placés en centre de rétention sauf si le Procureur des mineurs autorise la présence des enfants de l'étranger retenu lorsque les conditions du centre le permettent. Or, la loi espagnole ne protège pas un enfant qui se trouve en situation irrégulière de l'expulsion lorsque cette dernière est liée à celle de ses parents. Autrement dit, l'expulsion de la personne adulte à laquelle le mineur a été confié entrainerait l'expulsion simultanée de l'enfant confié. On doit enfin préciser que l'expulsion ne constitue pas une sanction d'ordre pénal en Espagne, bien qu'une interdiction de territoire pouvant aller jusqu'à 5 ans y est régulièrement associée.

En somme, une hypothétique reconnaissance de la pratique coutumière du " confiage » d'un mineur sénégalais à une personne adulte qui essaie elle aussi d'entrer clandestinement sur le territoire espagnol impliquerait l'application au mineur d'un traitement légal nettement moins protecteur que celui prévu pour les mineurs considérés comme non accompagnés. Par ailleurs, l'absence habituelle de documents officiels faisant preuve de cette pratique coutumière pourrait nous inciter à penser que derrière certains confiages pourraient se cacher des situations de traite ou exploitation de l'enfant concerné.

En guise de conclusion, nous pouvons et devons faire remarquer le déséquilibre existant entre, d'un côté, l'articulation dans le contexte sénégalais d'une circulation migratoire spécifique qui engendre différentes attentes et expectatives des acteurs impliqués (enfants migrants, parents, autres adultes concernés, etc.) et, de l'autre, la nécessité européenne, ou dans ce cas particulier espagnole, d'essayer d'encadrer cette réalité dans une logique normative. Or, si les normes nationales et européennes ne sont pas toujours capables de s'adapter et de tenir compte des différentes particularités et nuances d'une réalité sociale concrète (on accuse les Lois qui règlent le statut d'une personne étrangère de rester insensibles et d'ignorer les souffrances, les besoins et les envies légitimes de l'être humain concerné), ces carences législatives peuvent parfois avoir des effets positifs. Dans le cas des mineurs sénégalais qui voyagent vers le territoire des Îles Canaries, la nonreconnaissance de la pratique coutumière de «confiage» par les autorités espagnoles (consciemment ou par ignorance) épargne ces enfants migrants du régime des sanctions qui sera automatiquement appliqué aux migrants adultes qui les accompagnent. L'identification automatique de ces mineurs comme «non accompagnés » implique l'application d'un traitement légal ad hoc qui adoucit - à partir de l'incorporation de différents préceptes de la législation de protection de l'enfance - le côté sécuritaire propre aux normes du Droit des étrangers. L'autre question est de savoir si ce traitement spécifique que l'on applique aux mineurs non accompagnés est, dans la pratique, le plus adéquat et adapté à leurs besoins spécifiques de protection et à leurs objectifs migratoires.

Daniel Senovilla Hernàndez

Docteur en Droit Chercheur contractuel projet PUCAFREU MIGRINTER - UMR 6588 / CNRS dansenher@gmail.com

Elh Ousmane Cissé Doctorant en Géographie MIGRINTER - UMR 6588 CNRS / Université de Poitiers cissemane@yahoo.fr 


\section{Bibliographie :}

Adams, Adrian (1977) Le long voyage des gens du flewve, Paris, Maspéro, 226 p.

Bouilly, Emmanuelle (2008) Les enjeux féminins de la migration masculine: le collectif des femmes pour la lutte contre l'immigration clandestine de Thiaroye-SurMer, Politique africaine, $\mathrm{n}^{\circ} 109$, pp. 16-31.

Cisse, Elh Ousmane (2009) Étude de l'émigration des mineurs sénégalais: exemple le quartier de Hann à Dakar, Poitiers, Universitré de Poitiers, 103 p.

Mém. Master II : Géo. : Poitiers : 2009.

Gonin, Patrick ; Robin, Nelly (2009) Les routes migratoires par le Sénégal, in Bensâad, Ali (dir.), Le Maghreb à l'épreuve des migrations subsabariennes: immigration sur émigration, Paris, Karthala, pp. 137-167.

Iglesias Martinez, Juan ; Legaz Torres, Francisco (2009) Estudio sobre la migracion internacional de menores extranjeros no acompañados hacia las Islas Canarias. Perfil y expectativas, Madrid, Instituto Universitario de Estudios sobre Migraciones \& Fundacion Nuevo Sol, 188 p.
Robin, Nelly ; Senovilla Hernández, Daniel (2010) The migration of unaccompanied \& separated Senegalese children to Spain, in Senovilla Hernández, Daniel ; Touzenis, Kristina ; Kanics, Jyothi (dir.) Migrating alone: unaccompanied and separated children's migration to Europe, Paris, Éditions UNESCO, pp. 143154.

Traoré, Sadio (1994) Les modèles migratoires Soninké et Poular de la vallée du fleuve Sénégal, Revue Européenne des Migrations Internationales, vol. 10, $\mathrm{n}^{\circ} 3$, pp. 61-81.

Van Lindth, Timon; Meier, Franziska (2008) Migration des mineurs sénégalais : le traitement et la protection des mineurs étrangers non accompagnés en Espagne, en France et en Italie, Paris, OIMOrganisation Internationale pour les Migrations, Bureau régionale pour l'Afrique de l'Ouest., 143 p.

Willems, Ross (2008) Les fous de la mer : les migrants clandestins du Sénégal aux Îles Canaries en 2006, in Diop, Momar Coumba (dir), Le Sénégal des migrations mobilité, identités et sociétés, Paris, Karthala, pp. 277-303. 